\title{
FREE RADICAL DAMAGE: ROLE IN VARICELLA ZOSTER VIRUS INFECTION
}

\author{
Olga Volobuieva \\ Department of Infectious Diseases and Clinical Immunology, School of Medicine ${ }^{1}$ \\ Tetiana Liadova \\ Department of Infectious Diseases and Clinical Immunology, School of Medicine ${ }^{l}$
}

Mykola Popov

Department of Infectious Diseases and Clinical Immunology, School of Medicine ${ }^{l}$

Olga Sorokina

Department of Infectious Diseases and Clinical Immunology, School of Medicine ${ }^{1}$

Olena Ognivenko

Department of Infectious Diseases and Clinical Immunology, School of Medicine ${ }^{1}$

Diana Dorosh $\bowtie$

Department of Infectious Diseases and Clinical Immunology, School of Medicine ${ }^{1}$ diana.dorosh@karazin.ua

${ }^{1}$ V. N. Karazin Kharkiv National University

6 Svobody sq., Kharkiv, 06122, Ukraine

$\triangle$ Corresponding author

\begin{abstract}
Study of the role of lipid peroxidation and antioxidant system in patients with infectious diseases is of great interest to researchers. Numerous studies have led to a common understanding of their contribution to the pathogenesis of infectious diseases, as well as to the complications development. However, the state of lipid peroxidation and antioxidant protection in patients with chickenpox (varicella) has not been sufficiently studied which is important for the development of new targeted treatments.

The aim of the research - to assess the state of lipid peroxidation (LPO) and the antioxidant system (AOS) in the dynamics in patients with chickenpox, depending on the severity of the disease.

Materials and methods. We selected for participating in the study 240 chickenpox patients (142 women and 98 men) aged 18-40 years. Chickenpox was diagnosed using clinical, serological, and molecular genetic methods. The state assessment of LPO in chickenpox patients was based on the determination of serum diene conjugates (DC), malondialdehyde (MDA) levels and the total oxidative activity (TOA) of blood plasma.

Results. Study showed that the process of lipid peroxidation increases and the activity of the antioxidant system decreases in patients with chickenpox during the acute period of the disease. The extent of these processes becomes greater as the severity of chickenpox increases. A decrease in the activity of antioxidant enzymes and a disruption in the functioning of the glutathione system in patients with moderate to severe hypertension leads to disruption of protective mechanisms and, as a result, to an increase in the free radical chain reactions, the uncontrolled growth of which causes irreversible damage to the membranes of various cells, which underlies visceropathy in chickenpox patients.

Conclusions. Antioxidant system is activated in the acute period in patients with mild chickenpox. It is evidenced by an increase in the overall antioxidant activity of blood plasma $(p=0.045)$ and red blood cells ( $p=0.00087)$, in the activity of catalase $(p=0.001)$, superoxide dismutase ( $p=0.0093)$, glutathione peroxidase $(p=0.036)$, glutathione reductase of plasma and red blood cells, and an increase in the concentration of oxidized and reduced glutathione in blood. Lipid peroxidation is hyperactivated in the acute period in patients with a moderate and severe course of the disease and an excess amount of primary and secondary hydroperoxides of fatty acids accumulates in the blood. This is accompanied by indicates a developing imbalance between the oxidative and antioxidant systems in group of patients with moderate and severe chickenpox. Our findings confirm the usefulness of antioxidants in the treatment of chickenpox.

Keywords: chickenpox, lipid peroxidation, antioxidant system, glutathione system.
\end{abstract}

DOI: $10.21303 / 2504-5679.2021 .001990$ 


\section{Introduction}

In the past decade it became accepted that free radicals, lipid peroxidation and antioxidant defence play a role in various tissues damages, thus in certain infectious diseases as well. In particular, it was found that there is an intensification of the free radical oxidation of lipids and a deepening of antioxidant deficiency in the development process of salmonellosis [1], viral hepatitis [2, 3], pseudotuberculosis, yersiniosis [4], erysipelas [5], diphtheria [6] and infectious mononucleosis [7]. These changes correlate with the disease severity. Excessive accumulation of lipid peroxidation products in patients with an infection profile leads to a protracted course of the disease and the development of various complications $[8,9]$. However, there are still gaps in the study and determination of the contribution to the pathogenesis of chickenpox of lipid peroxidation and the antioxidant system.

The aim of the research was to assess the state of lipid peroxidation (LPO) and the antioxidant system (AOS) in the dynamics in patients with chickenpox, depending on the severity of the disease.

\section{Materials and methods}

In study, 240 chickenpox patients (142 women and 98 men) aged 18-40 years who were hospitalized in the community-serving non-profit organizations «Kharkiv Regional Clinical Hospital of Infectious Diseases» were examined from 2012 to 2019.

The study was conducted in accordance with the requirements of good clinical practice, the Council of Europe Convention on Human Rights and Biomedicine, the Helsinki Declaration of the World Medical Association and approved by the local ethics committee of the V. N. Karazin Kharkiv National University No. 6/12 21.05.2012. All patients signed informed consent for participating in the study.

Mild chickenpox infection was diagnosed in $82(34.2 \%)$ patients, moderate in 143 ones $(59.6 \%)$ and severe in $14(6.4 \%)$ patients. Chickenpox was diagnosed using clinical, serological, and molecular genetic methods. The state assessment of LPO in chickenpox patients was based on the determination of serum diene conjugates (DC), malondialdehyde (MDA) levels and the total oxidative activity (TOA) of blood plasma [10]. AOS status was determined based on the total antioxidant capacity (TAC) of blood plasma and red blood cells (RBCs), the activity of catalase, superoxide dismutase, glutathione peroxidase, erythrocyte glutathione reductase, blood glutathione peroxidase, blood plasma glutathione reductase, total glutathione, oxidized and reduced glutathione in blood plasma $[11,12]$. Examination of patients with chickenpox was carried out in the acute period of the disease, after normalization of body temperature and before hospital discharge.

All patients received standard therapy, which included antiviral drugs (acyclovir, valacyclovir), reosorbilact, antral, desloratadine, and antibacterial drugs.

Statistical analysis of the results was performed using the IBM SPSS Statistics statistical package (FacultyPack L/N: L-GLBC-99H6WQ) and Microsoft Excel (Office Home Business 2KB4Y-6H9DB-BM47K-749PV-PG3KT). Quantitative data are presented as mean values (M) and standard deviations (SD). For comparison of values between groups, Student's t-test and Wilcoxon test were used. To describe the correlation we used the Pirson's criterion. The critical significance level of statistical significance at the null hypothesis test was assumed to be 0.05 .

\section{Results}

Indicators of oxidative and antioxidant systems obtained in chickenpox patients are shown in Table 1. Analysis of the studied parameters showed that there was a statistically significant increase in content of primary (DC, $p<0.001$ ) and secondary (MDA, $p<0.001$ ) lipid peroxides in the blood and TOA of blood plasma $(p<0.001)$ as the severity of the disease increased in patients with chickenpox. Such changes in these patients indicated activation of LPO.

A high correlation $(r=0.62, p=0.012, r=0.67, p=0.027)$ was found between the content of DC and MDA in the blood, on the one hand, and the TOA of blood plasma, on the other hand, which indicated a close relationship between the increase in plasma oxidative activity and the formation of primary and secondary LPO products. 
Table 1

Indicators of lipid peroxidation and antioxidant system in patients with chickenpox $(\mathrm{M} \pm \mathrm{m})$

\begin{tabular}{|c|c|c|c|c|}
\hline \multirow[b]{2}{*}{ Indicator } & \multirow{2}{*}{$\begin{array}{c}\text { Control } \\
\text { group } \\
(n=30)\end{array}$} & \multicolumn{3}{|c|}{ Severity } \\
\hline & & $\begin{array}{l}\text { Mild course } \\
\quad(n=84)\end{array}$ & $\begin{array}{c}\text { Moderate } \\
\text { course }(n=143)\end{array}$ & $\begin{array}{l}\text { Severe course } \\
\quad(n=15)\end{array}$ \\
\hline Diene conjugates, $\mu \mathrm{mol} / \mathrm{L}$ & $1.2 \pm 0.02$ & $2.2 \pm 0.04 *$ & $3.6 \pm 0.03^{*}$ & $4.8 \pm 0.09^{*}$ \\
\hline Malondialdehyde, $\mu \mathrm{mol} / \mathrm{L}$ & $0.35 \pm 0.01$ & $1.5 \pm 0.038^{*}$ & $3.6 \pm 0.05^{*}$ & $4.9 \pm 0.06^{*}$ \\
\hline Total oxidative activity of plasma, $\%$ & $4.3 \pm 0.3$ & $6.4 \pm 0.4^{*}$ & $8.6 \pm 0.48^{*}$ & $11.6 \pm 0.6^{*}$ \\
\hline Total antioxidant capacity of plasma, $\%$ & $8.2 \pm 0.8$ & $11.6 \pm 1.2^{*}$ & $6.6 \pm 0.9^{*}$ & $4.0 \pm 0.5^{*}$ \\
\hline Total antioxidant capacity of red blood cells, $\%$ & $41.5 \pm 1.2$ & $49.6 \pm 1.3^{*}$ & $30.5 \pm 1.4^{*}$ & $23.6 \pm 1.8^{*}$ \\
\hline Catalase activity of red blood cell, $\mathrm{mmol} / \mathrm{s} \mathrm{mg}$ protein & $67.2 \pm 2.6$ & $75.4 \pm 1.8^{*}$ & $39.2 \pm 1.9^{*}$ & $25.6 \pm 1.5^{*}$ \\
\hline Superoxide dismutase activity of red blood cells, units/mg HB & $63.8 \pm 2.3$ & $70.6 \pm 1.7^{*}$ & $45.8 \pm 1.5^{*}$ & $35.8 \pm 1.6^{*}$ \\
\hline Total blood glutathione, $\mathrm{mmol} / \mathrm{L}$ & $990.0 \pm 9.4$ & $1216.5 \pm 7.5^{*}$ & $826.2 \pm 6.9^{*}$ & $613.5 \pm 5.7^{*}$ \\
\hline Oxidized blood glutathione, $\mathrm{mmol} / \mathrm{L}$ & $50.4 \pm 4.7$ & $92.5 \pm 6.0^{*}$ & $226.5 \pm 5.3^{*}$ & $268.5 \pm 6.4^{*}$ \\
\hline Reduced blood glutathione, $\mathrm{mmol} / \mathrm{L}$ & $940.5 \pm 8.3$ & $1020.2 \pm 8.6^{*}$ & $638.3 \pm 9.4^{*}$ & $350.4 \pm 3.8^{*}$ \\
\hline Erythrocyte glutathione peroxidase activity, $\mu \mathrm{mol} / \mathrm{s} \mathrm{mg}$ protein & $183.8 \pm 5.6$ & $194.5 \pm 6.5^{*}$ & $135.2 \pm 5.8^{*}$ & $107.5 \pm 4.5^{*}$ \\
\hline Plasma glutathione peroxidase activity, $\mu \mathrm{kat} / \mathrm{L}$ & $3.1 \pm 0.13$ & $5.0 \pm 0.16^{*}$ & $2.0 \pm 0.08^{*}$ & $1.2 \pm 0.05^{*}$ \\
\hline Plasma glutathione reductase activity, $\mu \mathrm{kat} / \mathrm{L}$ & $2.0 \pm 0.02$ & $3.1 \pm 0.06^{*}$ & $1.7 \pm 0.03 *$ & $0.8 \pm 0.02 *$ \\
\hline Erythrocyte glutathione reductase activity, $\mu \mathrm{mol} / \mathrm{s} \mathrm{mg}$ protein & $73.7 \pm 2.6$ & $98.7 \pm 1.9^{*}$ & $73.5 \pm 2.1^{*}$ & $57.6 \pm 1.8^{*}$ \\
\hline
\end{tabular}

Note: *-the significance of differences p-value compared with the previous group statistically significant on levels $p<0.05$

LPO activation was accompanied by activation of the antioxidant system in patients with mild chickenpox evidenced by a statistically significant increase in the TAC of blood plas$\mathrm{ma}(p=0.045)$ and RBCs $(p=0.00087)$, the activation of erythrocyte antiperoxidative enzymes, namely catalase $(p=0.001)$, superoxide dismutase $(p=0.0093)$, glutathione peroxidase $(p=0.036)$ and glutathione systems. An increase in the content of total, oxidized, and reduced glutathione as well as activation of the glutathione peroxidase in RBCs, plasma glutathione peroxidase and reductase were observed in blood in most patients. Therefore, activation of the AOS, which includes antioxidant enzymes (catalase, superoxide dismutase, glutathione peroxidase, glutathione reductase) and glutathione, occurs in response to increased lipid oxidation in patients with mild chickenpox. This contributed to the normalization of DC and MDA blood concentration on the 7-10 $0^{\text {th }}$ day of the disease. The activity of antioxidant enzymes remained elevated until the $15-20^{\text {th }}$ day of the disease.

A significant increase in blood concentration of DC and MDA, oxidative activity of blood plasma, decrease in antioxidant activity of blood plasma and red blood cells were observed in patients with moderate and severe course, which is associated with inhibition of the antioxidant enzymes activity of RBCs, namely catalase, superoxide dismutase, glutathione peroxidase and plasma glutathione reductase, and with the glutathione concentration decrease in blood.

The data above indicated that chickenpox patients with moderate and severe course of the disease developed a pronounced imbalance between the state of the oxidative and antioxidant systems, which was accompanied by an increase in the free radical oxidation of fatty acids and the accumulation of toxic DC and MDA increased concentration [7, 13]. A marked deficiency of glutathione, which normally inactivates free radicals [14], also developed and could have a negative impact on the course of the disease and its complications.

During the period of complex therapy in patients with moderate and severe chickenpox the content of DC and MDA and the oxidative activity of plasma gradually decreased as the clinical manifestations of the disease in the blood decreased. This process was accompanied by an increase in the antioxidant activity of plasma and red blood cells, the activity of catalase, superoxide dismutase, glutathione peroxidase and glutathione reductase in blood plasma and red blood cells. Normalization of these indicators occurred on the $20-30^{\text {th }}$ day. 


\section{Discussions}

LPO is one of the most important biological processes that constantly takes place in the human body. It is a part of the processes of adaptation, anti-infectious protection, removal of endoand exotoxins, tumour cells and tissue destruction, regulation of vascular tone, permeability of cell membranes and vascular wall, hemostasis, etc. $[15,16]$. Under normal conditions, LPO proceeds constantly in all cells at an extremely low level, which ensures the regulation of the structure and function of cell membranes $[17,18]$. The most reactive ones in this regard are the cells of the monocyte macrophage system, Kupffer liver cells, alveolar macrophages, macrophages of connective tissue, Langerhans cells, osteoclasts, glia astrocytes, which are activated by infection, antibodies, antigens, C-reactive protein, complement components [19, 20].

Granulocyte lysosomes contain such enzymes as NAD, NADPH oxidase, which is activated during phagocytosis and catalyzes the process of molecular oxygen reduction with the formation of oxygen with an unpaired electron. The intracellular enzyme superoxide dismutase binds this oxygen to hydrogen and hydrogen peroxide is formed, which is then cleaved by catalase and glutathione peroxidase to form hydroxide and hydroperoxide radicals that inactivate glutathione [21, 22]. Glutathione is converted into an oxidized form. It is converted to reduced glutathione under the influence of the enzyme glutathione reductase and ensures the binding of lipid hydroxides.

Glutathione peroxidase and glutathione transferase are capable of reducing hydroperoxides - groups of oxidized phospholipids directly in cell membranes, without prior hydrolyzing them by phospholipase or free fatty acids [23, 24].

Consequently, glutathione and enzymes of its metabolism are one of the most important universal defence mechanisms and the central link in the homeostatic system of the body, which play a primary role in the formation of the body's resistance to aggressive factors $[25,26]$.

Glutathione is formed in the liver and contains glutamic acid, cysteine and glycine. Having the $\mathrm{SH}$ functional group, glutathione is involved in redox reactions as a hydrogen donor and passes from the reduced to the oxidized form [10].

Antioxidant enzymes normally maintain free radical oxidation at a safe level. They restore oxygen to less active forms and also inhibit the formation of $\mathrm{HO}^{-}, \mathrm{HO}_{2}-\mathrm{H}_{2} \mathrm{O}_{2}$ and destroy excess amount of lipid hydroxide [27, 28]. Given that high levels of $\mathrm{NO}$ and $\mathrm{H}_{2} \mathrm{O}_{2}$ were observed in patients with herpes zoster infection, search for the methods of the antioxidant defence disorders correction in patients with chickenpox seems promising [29].

The LPO system is well balanced and operates on a feedback basis, since LPO products inhibit the activity of antioxidant enzymes.

However, the activation of LPO in mild chickenpox patients is accompanied by the increased activity of catalase, superoxide dismutase, glutathione peroxidase, glutathione reductase and the concentration of reduced glutathione in the blood, which binds and oxidizes primary and secondary lipid hydroxides. It contributes to a significant acceleration of activity restoration processes of antioxidant enzymes antioxidant activity of plasma and RBCs.

LPO activation in patients with moderate to severe chickenpox leads to a deep suppression of antiperoxidation system activity. Normalization of this system proceeds slowly over a long period of time.

The decrease in the activity of catalase, superoxide dismutase, glutathione peroxidase, glutathione reductase in blood plasma and erythrocytes, and the concentration of total and reduced glutathione in blood observed in patients with moderate and severe chickenpox indicates a deep impairment of the functioning of various parts of the antioxidant system. This contributes to the increased formation of reactive oxygen species, hydroxide radicals, hydrogen peroxide in cells, causing oxidation of unsaturated fatty acids on cell membranes and the formation of primary and secondary lipid hydroperoxides, which have a toxic effect not only on varicella zoster virus, but also on other cellular structure.

Lipid peroxides are highly reactive and can suppress the activity of catalase, superoxide dismutase, glutathione peroxidase, glutathione reductase. It can also cause the destruction of SH-containing compounds, DNA depolymerization, vasospasm, endothelium damage and increase the 
permeability of cell membranes and platelet aggregation [30]. It was found that the balance of oxygen metabolism is compromised in cells infected by HSV-1, namely there were the increased levels of the lipid peroxidation product MDA [31].

Oxidized glutathione also has toxic properties. It accumulates in tissues in patients with moderate to severe chickenpox as a result of a decrease in glutathione reductase activity. Oxidized glutathione inactivates membrane adenosine triphosphatase, hexokinase, glucose-6-dehydrogenase, inhibits phosphorylation and nuclear RNA synthesis, and inhibits protein synthesis due to the formation of protein-thiol-glutathione crosslinks [7, 15].

Our study showed that LPO rate increases and AOS activity decreases in patients with chickenpox in the acute period of the disease. These processes become more pronounced as the severity of the disease increases. A decrease in the activity of antioxidant enzymes and a disruption in the functioning of the glutathione system in chickenpox patients with moderate and severe course leads to a violation of the protective mechanisms.

As a result, an increase in the free radical chain reactions takes place and its uncontrolled growth causes irreversible damage to the membranes of various cells, which underlies visceropathies in chickenpox patients.

Thus, adaptive capacity of antioxidant enzymes is reduced in patients with chickenpox. This may be a consequence of primary or secondary enzyme defect, due to the toxic effect of reactive oxygen species and lipid hydroperoxides on the enzymes. Our findings confirm the usefulness of antioxidants in the treatment of chickenpox.

Study limitations. The present research was aimed at studying only lipid peroxidation and antioxidant system in chickenpox patients and their contribution to the pathogenesis of disease. The level of quality of life was not discussed.

Prospects for further research are aimed at assessment of clinical and immunological features of chickenpox of the studied groups. It is also necessary to draw up to evaluate the impact of disease on the quality of life.

\section{Conclusions}

Antioxidant system is activated in the acute period in patients with mild chickenpox. It is evidenced by an increase in the overall antioxidant activity of blood plasma $(p=0.045)$ and red blood cells $(p=0.00087)$, in the activity of catalase $(p=0.001)$, superoxide dismutase $(p=0.0093)$, glutathione peroxidase $(p=0.036)$, glutathione reductase of plasma and red blood cells, and an increase in the concentration of oxidized and reduced glutathione in blood.

Lipid peroxidation is hyperactivated in the acute period in patients with a moderate and severe course of the disease and an excess amount of primary and secondary hydroperoxides of fatty acids accumulates in the blood. This is accompanied by a decrease in the antioxidant activity of blood plasma and erythrocytes, the activity of catalase, superoxide dismutase, glutathione peroxidase, glutathione reductase blood plasma and red blood cells and indicates a developing imbalance between the oxidative and antioxidant systems in group of patients with moderate and severe chickenpox.

\section{Conflict of interests}

The authors declare that they have no conflict of interests.

\section{Financing}

The study was done without financial support.

\section{References}

[1] Schürmann, N., Forrer, P., Casse, O., Li, J., Felmy, B., Burgener, A.-V. et. al. (2017). Myeloperoxidase targets oxidative host attacks to Salmonella and prevents collateral tissue damage. Nature Microbiology, 2 (4). doi: http://oi.org/10.1038/ nmicrobiol.2016.268

[2] Alavian, S. M., Showraki, A. (2016). Hepatitis B and its Relationship With Oxidative Stress. Hepatitis Monthly, 16 (9). doi: http://doi.org/10.5812/hepatmon.37973 
[3] Darenskaya, M. A., Grebenkina, L. A., Sholokhov, L. F., Rashidova, M. A., Semenova, N. V., Kolesnikov, S. I., Kolesnikova, L. I. (2016). Lipid Peroxidation Activity in Women with Chronic Viral Hepatitis. Free Radical Biology and Medicine, 100, S192. doi: http://doi.org/10.1016/j.freeradbiomed.2016.10.525

[4] Isberg, R. R. (2017). Internalization of Microbial Pathogens by Integrin Receptors and the Binding of the Yersinia pseudotuberculosis Invasin Protein. Integrins - The Biological Problems. CRC Press, 197-216.

[5] Emene, P. E., Kravchenko, I. E., Aibatova, G. I., Rizvanov, A. A. (2015). Antioxidant system gene polymorphism in patients with erysipelas and their role in the development of the disease. Genes and cells, 10 (4), 118-122.

[6] Mayer, K., Mundigl, O., Kettenberger, H., Birzele, F., Stahl, S., Pastan, I., Brinkmann, U. (2019). Diphthamide affects selenoprotein expression: Diphthamide deficiency reduces selenocysteine incorporation, decreases selenite sensitivity and pre-disposes to oxidative stress. Redox Biology, 20, 146-156. doi: http://doi.org/10.1016/j.redox.2018.09.015

[7] Shustval, M. F., Lyadova, T. I., Volobueva, O. V., Pavlikova, K. V., Gamilovskaya, A. P. (2018). State of lipid peroxidation and oxidant system in patients with infectious mononucleosis. Bulletin of the VN Kharkiv National University Karazin. Series «Medicine», 36, 33-40. doi: http://doi.org/10.26565/2313-6693-2018-36-05

[8] Bwititi, P., Chinkwo, K. (2016). Oxidative stress markers in infectious respiratory diseases: current clinical practice. International Journal of Research in Medical Sciences, 1802-1813. doi: http://doi.org/10.18203/2320-6012.ijrms20161727

[9] Ramana, K. V., Srivastava, S., Singhal, S. S. (2017). Lipid Peroxidation Products in Human Health and Disease 2016. Oxidative Medicine and Cellular Longevity, 2017, 1-2. doi: http://doi.org/10.1155/2017/2163285

[10] Tuktanov, N. V., Kichigin, V. A. (2013). Kichigin features lipid peroxidation of thyroid dysfunction. Bulletin of the Chuvash University, 3, 55-560.

[11] Karpishchenko, L. I. (2002). Reference book. Medical laboratory equipment. Saint Petersburg: Intermedika, 245.

[12] Kolesnikova, L. I., Darenskaya, M. A., Rashidova, M. A., Sholokhov, L. F., Grebenkina, L. A., Vanteeva, O. A. (2016). Lipid Peroxidation State in Women of Reproductive Age with Acute Form of Viral Hepatitis. Annals of the Russian Academy of Medical Sciences, 71 (1), 11-15. doi: http://doi.org/10.15690/vramn525

[13] Putilina, F. E.; Prokhorova, M. I. (Ed.) (1982). Determination of glutathione reductase activity. Methods of biochemical research, 181-186.

[14] Poprac, P., Jomova, K., Simunkova, M., Kollar, V., Rhodes, C. J., Valko, M. (2017). Targeting Free Radicals in Oxidative Stress-Related Human Diseases. Trends in Pharmacological Sciences, 38 (7), 592-607. doi: http://doi.org/10.1016/ j.tips.2017.04.005

[15] Polozova, E. I., Trokhina, I. E., Kurkina, N. V., Gorshenina, E. I. (2017). Estimation of efficiency of application of combined therapy in complex treatment of duodenal ulcer. Modern problems of science and education, 3, 67.

[16] Saveris, M. J., Mayakova, E. I. (2018). Estimation of efficiency of application of combined therapy in complex treatment of duodenal ulcer. Tomorrow's Medicine, 43-44.

[17] Gaschler, M. M., Stockwell, B. R. (2017). Lipid peroxidation in cell death. Biochemical and Biophysical Research Communications, 482 (3), 419-425. doi: http://doi.org/10.1016/j.bbrc.2016.10.086

[18] Pawluk, H., Pawluk, R., Robaczewska, J., Kędziora-Kornatowska, K., Kędziora, J. (2017). Biomarkers of antioxidant status and lipid peroxidation in elderly patients with hypertension. Redox Report, 22 (6), 542-546. doi: http://doi.org/10.1080/ 13510002.2017.1372072

[19] Bhattacharya, S. (2015). Reactive oxygen species and cellular defense system. Free radicals in human health and disease. New Delhi: Springer, 17-29. doi: http://doi.org/10.1007/978-81-322-2035-0_2

[20] Conti, V., Corbi, G., Simeon, V., Russomanno, G., Manzo, V., Ferrara, N., Filippelli, A. (2015). Aging-related changes in oxidative stress response of human endothelial cells. Aging Clinical and Experimental Research, 27 (4), $547-553$. doi: http://doi.org/10.1007/s40520-015-0357-9

[21] Dua, A., Kaur, N., Gupta, P., Mittall, A., Gupta, S. K. (2017). Oxidative stress induced cell damage and antioxidant enzyme response in human lymphocytes. International Journal of Pharmaceutical \& Biological Archive, 8, 33-39.

[22] Ighodaro, O. M., Akinloye, O. A. (2018). First line defence antioxidants-superoxide dismutase (SOD), catalase (CAT) and glutathione peroxidase (GPX): Their fundamental role in the entire antioxidant defence grid. Alexandria Journal of Medicine, 54 (4), 287-293. doi: http://doi.org/10.1016/j.ajme.2017.09.001

[23] Allocati, N., Masulli, M., Di Ilio, C., Federici, L. (2018). Glutathione transferases: substrates, inihibitors and pro-drugs in cancer and neurodegenerative diseases. Oncogenesis, 7 (1). doi: http://doi.org/10.1038/s41389-017-0025-3

[24] Mead, J. F., Stein, R. A., Wu, G. S. (2019). Metabolic Fate of Peroxidation Products. Cellular Antioxidant Defense Mechanisms. CRC Press, 89-102. doi: http://doi.org/10.1201/9780429289286-6

[25] Griffiths, H. R., Gao, D., Pararasa, C. (2017). Redox regulation in metabolic programming and inflammation. Redox Biology, 12, 50-57. doi: http://doi.org/10.1016/j.redox.2017.01.023 
[26] Sies, H. (2017). Hydrogen peroxide as a central redox signaling molecule in physiological oxidative stress: Oxidative eustress. Redox Biology, 11, 613-619. doi: http://doi.org/10.1016/j.redox.2016.12.035

[27] Sies, H., Berndt, C., Jones, D. P. (2017). Oxidative Stress. Annual Review of Biochemistry, 86 (1), 715-748. doi: http://doi.org/ 10.1146/annurev-biochem-061516-045037

[28] Sies, H. (2015). Oxidative stress: a concept in redox biology and medicine. Redox Biology, 4, 180-183. doi: http://doi.org/ 10.1016/j.redox.2015.01.002

[29] Nasiri, S., Hedayati, M., Riahi, S., Robati, R., Khazan, M. (2018). Elevated serum nitric oxide and hydrogen peroxide levels as potential valuable predictors of herpes zoster. Asian Pacific Journal of Tropical Medicine, 11 (6), 381. doi: http://doi.org/ $10.4103 / 1995-7645.234766$

[30] Muriel, P. (2017). Peroxidation of lipids and liver damage. Oxidants, antioxidants and free radicals. CRC Press, $237-257$. doi: http://doi.org/10.1201/9780203744673-14

[31] Georgieva, A., Vilhelmova, N., Muckova, L., Tzvetanova, E., Alexandrova, A., Mileva, M. (2017). Alterations in oxidative stress parameters in MDBK cells, infected by herpes simplex virus-1. Comptes rendus de l'Académie bulgare des Sciences, 70 (5).

Received date 31.05.2021

Accepted date 27.07.2021

Published date 30.07.2021
(C) The Author(s) 2021

This is an open access article under the Creative Commons CC BY license

How to cite: Volobuieva, O., Liadova, T., Popov, M., Sorokina, O., Ognivenko, O., Dorosh, D. (2021). Free radical damage: role in varicella zoster virus infection. EUREKA: Health Sciences, 4, 41-47. doi: http://doi.org/10.21303/2504-5679.2021.001984 